\title{
Análisis descriptivo del concurso de ingreso al ciclo de destinación y formación de cirujanos dentistas entre los años 2009 y 2018.
}

\section{Descriptive analysis of the admission process to the destination and training cycle of dental surgeons between 2009 and 2018.}

\author{
Karla Moscoso-Matus ${ }^{1}$; Claudia Véliz²*; Rosario García-Huidobro2; Cynthia Cantarutti²; \\ Daniela Guzmán; Juan Pablo Vargas²
}

\begin{abstract}
1. Escuela de Odontología, Facultad de Medicina, Pontificia Universidad Católica de Chile, Santiago, Chile. | Fundación Sonrisas, Santiago, Chile. 2. Escuela de Odontología, Facultad de Medicina, Pontificia Universidad Católica de Chile, Santiago, Chile.
\end{abstract}

* Correspondencia Autor: Claudia Véliz | Direccion: Av. Vicuña Mackenna, 4860, Campus San Joaquín, comuna de San Joaquín | Teléfono: +56 96308 0808 | E-Mail: cvelizp@uc.cl

Trabajo recibido el 03/04/2020.

Trabajo revisado 09/09/2020

Aprobado para su publicación el 15/09/2020

\begin{abstract}
RESUMEN
Introducción. Desde 1955 existe en Chile una estrategia de recursos humanos destinada a llevar profesionales de la salud a zonas rurales o de difícil acceso, denominada ciclo de destinación y formación (ex generales de zona). Existe poca información sobre las características de los postulantes a él. Esta investigación tiene como objetivo describir las postulaciones para el ingreso al ciclo entre los años 2009 al 2018. Metodología: Se obtuvieron los datos del ciclo desde la web del Ministerio de Salud, los que fueron completados con datos de la Superintendencia de Salud y web del Ministerio de Educación. Los datos se analizaron utilizando el software SPSS. Resultados: Se analizaron 10 concursos de ingreso al ciclo con un total de 1.689 postulantes, que representan $13,8 \%$ de cirujanos dentistas recién titulados entre años 2009-2018. El $60 \%$ de los postulantes son mujeres, pero las postulaciones de hombres son más efectivas $(p=0,038) .5$ universidades concentran $63 \%$ de las postulaciones y el $86 \%$ de las plazas $(p<0,001)$. El offset se ha mantenido constante. Discusión: Existe poca investigación del tema y resulta de interés analizar las diferencias entre la efectividad de las postulaciones según sexo y universidad de origen, así como las motivaciones para postular.
\end{abstract}

PALABRAS CLAVE

Políticas públicas; Odontología; Equipos de trabajo en salud.

Int. J. Inter. Dent Vol. 14(2); 135-139, 2021.

\section{ABSTRACT}

Introduction. Since 1955, a human resources strategy has existed in Chile aiming to insert health professionals into rural or difficult-to-reach areas, called the EDF destination and training cycle (formerly, zone generals). There is little information regarding recent graduates entering this cycle and the factors conditioning their application. Therefore, this study aims to describe the cohorts that entered the EDF cycle between 2009 and 2018. Methodology: Data from the EDF application processes were obtained from the website of the Ministry of Health and completed with the records from the Superintendence of Health. Information on the total number of graduates from each Chilean university was obtained from the Ministry of Education website. The data were analyzed using the SPSS IBM Statistic v24 software. Results: Ten application processes for entering the EDF cycle were analyzed, encompassing a total of 1,689 applicants, representing $13.8 \%$ of the newly registered dental surgeons between 20092018. Even though $60 \%$ of the applicants are female, the applications from men are more effective $(p=0,038)$. Five universities concentrate $63 \%$ of the applications and $86 \%$ of the places $(p<0.001)$. The offset has remained constant. Discussion: There is little research on this subject and it would be interesting to analyze the reasons behind the differences between the effectiveness of the applications according to sex and university of origin, as well as the motivations of the applicants to apply.

KEY WORDS

Public policy; Dentistry; Health workforce.

Int. J. Inter. Dent Vol. 14(2); 135-139, 2021. 


\section{INTRODUCCIÓN}

Las dificultades para reclutar y mantener al personal de salud en zonas rurales han sido descritas por la Organización Mundial de la Salud, como posibles causas de deficientes resultados de salud en esas mismas áreas ${ }^{(1,2)}$. Por esta razón, se han realizado esfuerzos para mejorar los indicadores de salud de estas zonas.

Existen estudios sobre intervenciones para incrementar el reclutamiento y la retención de médicos de atención primaria de salud (APS), donde se concluye que hay débil evidencia de mejoras en la incorporación de médicos de APS con el reclutamiento en zonas rurales de estudiantes de medicina o de médicos extranjeros (con facilidades para la obtención de visa de trabajo) y la formación de postgrado en áreas rurales ${ }^{(3)}$. Asimismo, existe poca evidencia sobre los incentivos financieros para estudios de pre o post grado y, debido a la calidad metodológica de los estudios, no es posible obtener conclusiones sobre esquemas de retención y reingreso, reclutadores especializados e iniciativas de apoyo emocional(3).

En Chile, la estrategia de reclutamiento de profesionales de la salud para sectores rurales corresponde a una política de salud pública de larga data, que surge el año 1955 al amparo de la Ley 15.076 con el nombre de General de Zona (GZ). Esta política, tenía el objetivo de atraer médicos, odontólogos, bioquímicos y químico farmacéuticos a zonas rurales o de difícil acceso, ofreciéndoles beneficios económicos, acceso a especialización y carrera funcionaria. Esta forma de reclutamiento de recurso humano aseguraba la presencia de profesionales en la red de salud y la formación de capital humano avanzado(4).

La estrategia de GZ fue modernizada el año 2000, a través de la Ley 19.664, adquiriendo sus actuales características ${ }^{(5)}$. Al día de hoy, el ciclo de Etapa de Destinación y Formación (EDF) ex GZ permite la incorporación de médicos cirujanos y cirujanos dentistas a establecimientos de mediana o baja complejidad del sistema público de salud en la etapa de destinación (ED); mientras que en la etapa de formación (EF) les ofrece a los profesionales acceder a un cupo de especialización en alguna de las universidades en convenio(5).

Los profesionales en la ED se desempeñan en distintos establecimientos denominados plazas, durante un periodo de mínimo 3 y máximo de 6 años. El ingreso al ciclo sólo es posible cuando se pertenece a la última promoción de titulados del año en curso. Para ello, los postulantes deben acreditar su desempeño en distintas áreas, obteniendo un puntaje con el cual se elabora un ranking ${ }^{(6,7)}$. La adjudicación de las plazas se realiza en un llamado público ("a viva voz") en el que, de acuerdo al ranking obtenido, los postulantes pueden escoger alguna de las plazas ofrecidas en ese concurso ${ }^{(6,7)}$. Una vez terminada la ED los profesionales pueden postular a la EF, en la que podrán estudiar una especialidad, mediante un concurso de similares características al de ingreso al ciclo ${ }^{(6,7)}$.

Existe poca evidencia actualizada respecto a la efectividad de este programa y la evaluación que realizó el Ministerio de Hacienda el año 2018 no fue positiva en los ámbitos de diseño, implementación, eficiencia y resultados ${ }^{(8)}$. Y, por otra parte, la mayoría de los estudios existentes se refieren a los concursos de médicos cirujanos y tratan sobre la percepción y satisfacción de los profesionales que ingresan a la $\operatorname{ED}^{(9,10)}$, la cantidad de años que permanecen en ella y la especialidad que eligen en la $\mathrm{EF}^{(4)}$. Se encontró sólo un estudio que analizó el concurso de cirujano dentistas, el que describió los concursos de los años 2003 a $2009^{(6)}$

Siendo esta una importante política pública de captación y formación de personal para APS, es necesario conocer más sobre su comportamiento, es por esto que el objetivo de esta investigación fue describir el resultado de las postulaciones para el ingreso de cirujano dentistas de la última promoción a la ED del ciclo EDF, entre los años 2009 a 2018.

\section{MATERIAL Y MÉTODO}

Para acceder a la información, se solicitó a la Subsecretaría de Redes Asistenciales la información de los resultados de los últimos 10 años de concurso para ingresar al ciclo de destinación y formación de cirujano dentistas (2009 a 2018), vía Ley de Acceso a la Información de la Administración del Estado. La información fue entregada por medio de la publicación de un banner en la página web del Ministerio de Salud y contenía el nombre y apellidos de los postulantes, puntajes según rubro, resultado de la postulación al concurso, el ranking de cada postulante y el tipo de plaza obtenida, así como las bases de cada concurso.

Para completar la información se accedió al Registro Nacional de Prestadores Individuales de la Superintendencia de Salud, obteniéndose así la universidad de egreso, sexo y fecha de nacimiento de cada postulante. Fueron excluidos los postulantes que no contaban con la información completa.

Además, se obtuvo la información de número total de cirujanos dentistas titulados de universidades chilenas entre los años 2009 a 2018, través del portal www.mifuturo.cl del Ministerio de Educación (MINEDUC) y se compararon con la cantidad de postulantes a la ED en el mismo periodo.

La edad de cada postulante fue calculada utilizando la fecha de nacimiento y la fecha del cierre de cada concurso (31 de enero de cada año). Para cada concurso se calculó el offset (razón entre total de plazas ofrecidas y ranking del último aceptado).

\section{Análisis estadístico:}

Para medir asociación entre el resultado de postulación y las variables sexo, edad y universidad de origen, se utilizó las pruebas Chi cuadrado y $\mathrm{T}$ Student según el tipo de variable. Las pruebas fueron realizadas utilizando el software IBM SPSS Statistics v24.

Esta investigación cuenta con la aprobación de la Unidad de Ética y Seguridad de Investigación de la Facultad de Medicina de la Pontificia Universidad Católica de Chile, ID 181217005.

\section{RESULTADOS}

Entre los años 2009 y 2018 se ejecutaron 10 concursos de ingreso al EDF, con un total de 1.691 postulantes (se excluyeron dos individuos que no tenían sus registros completos, quedando una muestra de 1.689 postulantes, con una media de $168,9 \pm 31,8$ por año).

El $60 \%$ de los postulantes eran mujeres y un $40 \%$ hombres, con una edad promedio de 25,4 años $( \pm 2,01)$. Existió una diferencia estadísticamente significativa entre la edad de mujeres 25,3 años $( \pm$ 2 ) y hombres 25,5 años $( \pm 2)(p=0,02)$, aunque esta diferencia no es relevante para el análisis de los datos.

De un total de 12.281 titulados en el período analizado, 1.691 postularon a los diferentes concursos, lo que equivale a un $13,77 \%$ (14,2\% hombres; $13,5 \%$ mujeres) (tabla № 1 ).

La universidad con mayor cantidad de postulantes en relación a los titulados fue la Universidad de Concepción (38\%), seguida por la Universidad de La Frontera (31,4\%), Universidad de Chile (26,9\%), la Universidad Austral de Chile $(22,2 \%)$ y la Universidad del Desarrollo $(17,1 \%)$ (tabla $\left.\mathrm{N}^{\circ} 2\right)$. En relación al número de postulaciones exitosas (logra una plaza) respecto del total de titulados, destacó la Universidad de Chile $(16,8 \%)$ y la Universidad de Concepción (10,4\%) (tabla №2).

En estos 10 concursos se ofrecieron un total de 392 plazas, de las que 388 fueron adjudicadas, con un promedio de 38,8 $\pm 6,2$ adjudicaciones por año (tabla $\left.\mathrm{N}^{\circ} 1\right)$.

Tabla 1: Relación entre titulados, postulantes y adjudicantes en periodo 2009-2018.

\begin{tabular}{l|c|c|c|c|c}
\hline & Femenino & $\%$ & Masculino & $\%$ & Total \\
\hline Titulados & 7.527 & $61 \%$ & 4.754 & $39 \%$ & 12.281 \\
\hline Postulantes & 1.015 & $60 \%$ & 676 & $40 \%$ & 1.691 \\
\hline Adjudicados & 215 & $55,4 \%$ & 173 & $44,5 \%$ & 388 \\
\hline
\end{tabular}

Los cirujanos dentistas recién egresados que postularon en el periodo evaluado pertenecen a 21 universidades chilenas y 2 extranjeras (tabla $\mathrm{N}^{\circ} 3$ ).

En relación a las universidades de origen, existen 5 instituciones que en conjunto concentran el $63 \%$ de las postulaciones y al $86 \%$ de las plazas adjudicadas (figuras $\mathrm{N}^{0} 1$ y №2). Existió una asociación estadísticamente significativa entre la universidad de origen y el resultado de postulación $(p<0,001)$.

En relación a los puntajes promedios (promedio 75,2 $(66,6-79,6)$ ), máximos (promedio 92,6 $(90,1-98,2))$ y de corte (promedio 82,3 $(73,6-$ $86,7)$ ), no se observó variación en los diferentes años de concurso, salvo el año 2014 en el que hubo una baja en los puntajes promedio y de corte.

Del total de postulantes que accedieron a una plaza, 215 fueron mujeres y 163 hombres, sin embargo los hombres alcanzaron una mayor efectividad en su postulación, ya que accedieron a una plaza en mayor proporción que las mujeres, $26 \%$ y $21 \%$ respectivamente $(p=0,038)$.

En general el offset se ha mantenido constante en los 10 años de concurso, salvo el año 2014 en el que se observó un aumento importante (offset $=5,3$ ) lo que coincide con el único concurso del período evaluado en el que no se adjudicaron todas las plazas ofrecidas (figura $N^{\circ} 3$ ). 
Tabla 2: Relación entre total de titulados, postulantes y adjudicaciones en período 2009-2018 por universidad.

\begin{tabular}{|c|c|c|c|c|c|}
\hline \multirow{2}{*}{$\begin{array}{l}\text { Universidad de } \\
\text { Origen }\end{array}$} & \multirow{2}{*}{$\begin{array}{l}\text { Total de } \\
\text { Titulados }\end{array}$} & \multicolumn{2}{|c|}{$\begin{array}{c}\text { Total } \\
\text { Postulantes }\end{array}$} & \multicolumn{2}{|c|}{$\begin{array}{c}\text { Total } \\
\text { Adjudicados }\end{array}$} \\
\hline & & $\mathbf{N}^{\circ}$ & $\%^{1}$ & $\mathbf{N}^{\circ}$ & $\%^{2}$ \\
\hline $\begin{array}{l}\text { U. San } \\
\text { Sebastián }\end{array}$ & 1.905 & 192 & $10,10 \%$ & 22 & $1,20 \%$ \\
\hline U. Mayor & 1.271 & 77 & $6,10 \%$ & 5 & $0,40 \%$ \\
\hline $\begin{array}{l}\text { U. del } \\
\text { Desarrollo }\end{array}$ & 1.237 & 212 & $17,10 \%$ & 59 & $4,80 \%$ \\
\hline $\begin{array}{l}\text { U. Andrés } \\
\text { Bello }\end{array}$ & 1.221 & 64 & $5,20 \%$ & 5 & $0,40 \%$ \\
\hline U. de Chile & 829 & 223 & $26,90 \%$ & 139 & $16,80 \%$ \\
\hline $\begin{array}{l}\text { U. de } \\
\text { Concepción }\end{array}$ & 689 & 262 & $38,00 \%$ & 72 & $10,40 \%$ \\
\hline U. de Talca & 648 & 87 & $13,40 \%$ & 7 & $1,10 \%$ \\
\hline $\begin{array}{l}\text { U. de Los } \\
\text { Andes }\end{array}$ & 633 & 30 & $4,70 \%$ & 0 & $0,00 \%$ \\
\hline $\begin{array}{l}\text { U. de } \\
\text { Valparaíso }\end{array}$ & 607 & 118 & $19,40 \%$ & 17 & $2,80 \%$ \\
\hline $\begin{array}{l}\text { U. de La } \\
\text { Frontera }\end{array}$ & 577 & 181 & $31,40 \%$ & 42 & $7,30 \%$ \\
\hline U. Finis Terrae & 549 & 19 & $3,50 \%$ & 0 & $0,00 \%$ \\
\hline $\begin{array}{l}\text { U. de } \\
\text { Antofagasta }\end{array}$ & 503 & 35 & $7,00 \%$ & 1 & $0,20 \%$ \\
\hline $\begin{array}{l}\text { U. Diego } \\
\text { Portales }\end{array}$ & 492 & 57 & $11,60 \%$ & 4 & $0,80 \%$ \\
\hline $\begin{array}{l}\text { U. Austral de } \\
\text { Chile }\end{array}$ & 388 & 86 & $22,20 \%$ & 13 & $3,40 \%$ \\
\hline $\begin{array}{l}\text { P. Universidad } \\
\text { Católica de } \\
\text { Chile }\end{array}$ & 237 & 23 & $9,70 \%$ & 0 & $0,00 \%$ \\
\hline $\begin{array}{l}\text { U. Pedro de } \\
\text { Valdivia }\end{array}$ & 146 & 6 & $4,10 \%$ & 1 & $0,70 \%$ \\
\hline $\begin{array}{l}\text { U. Autónoma } \\
\text { de Chile }\end{array}$ & 125 & 1 & $0,80 \%$ & 0 & $0,00 \%$ \\
\hline $\begin{array}{l}\text { U. de Viña Del } \\
\text { Mar }\end{array}$ & 81 & 4 & $4,90 \%$ & 0 & $0,00 \%$ \\
\hline U. Bolivariana & 63 & 5 & $7,90 \%$ & 1 & $1,60 \%$ \\
\hline U. del Mar & 49 & 4 & $8,20 \%$ & 0 & $0,00 \%$ \\
\hline U. Arturo Prat & 31 & 1 & $3,20 \%$ & 0 & $0,00 \%$ \\
\hline
\end{tabular}

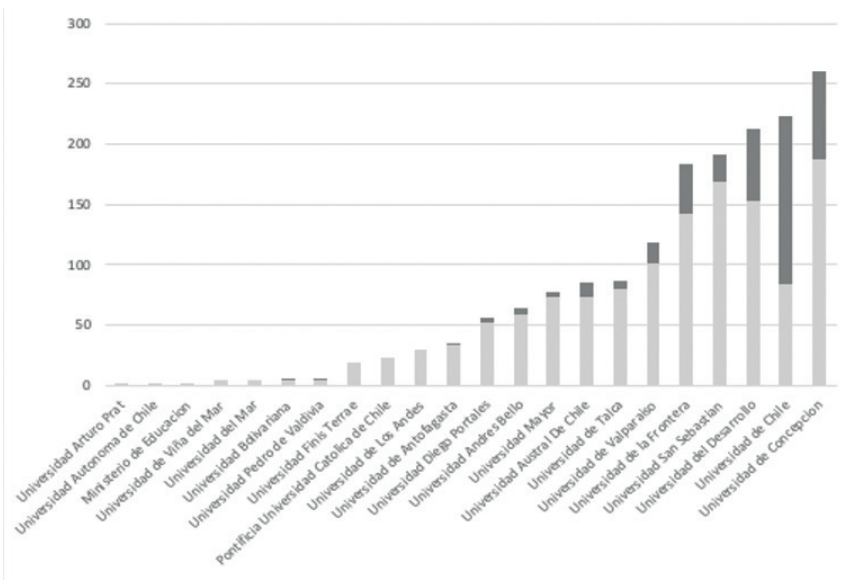

- No adjudica plaza InSi a afudica plaza

$\overline{\text { Figura 1. Postulantes que adjudica y no adjudica plaza por universidad }}$ de origen.

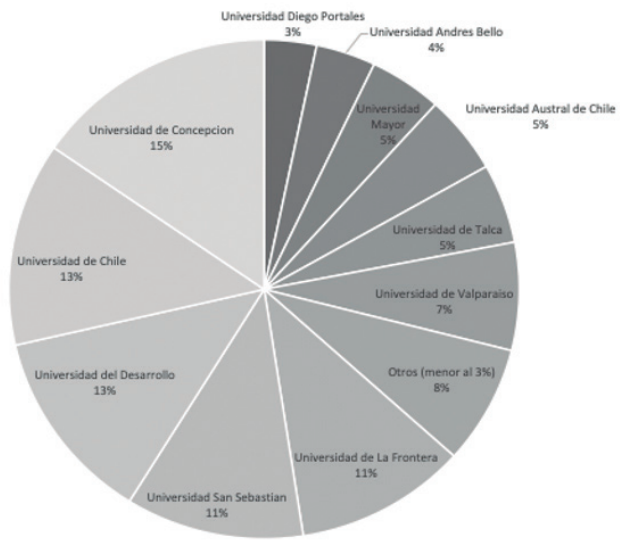

Figura 2. Proporción de postulantes por universidad de origen.

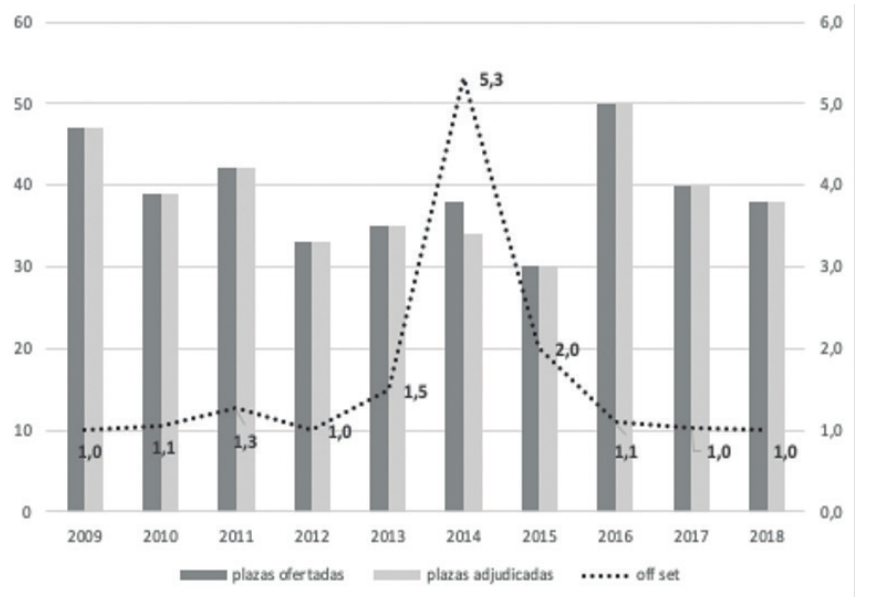

Figura 3. Variación de puntajes según plazas ofertadas y adjudicada, por año de concurso.

\section{DISCUSIÓN}

En los 65 años de implementación de esta política pública, la investigación asociada al concurso de cirujano dentistas ha sido casi nula, por lo que se cuenta con muy poca información sobre las características de los postulantes y los aspectos que deben potenciar para tener una mayor posibilidad de adjudicarse una plaza. Además, al no contar con información descriptiva sobre el comportamiento de las diferentes postulaciones por año del concurso (postulaciones/ año), resulta difícil la implementación de estrategias para optimizar los resultados y cumplir los objetivos de esta política pública.

En todas las postulaciones/año analizadas se observó una mayor proporción de mujeres postulantes, esta característica se mantiene constante desde el año 2003. ${ }^{(6)}$, y es concordante con la cantidad de mujeres y hombres que se titulan de Cirujano Dentistas, que para el periodo $2009-2018$ fue de $61 \%$ y $39 \%$, respectivamente ${ }^{(11)}$. Esta mayor participación femenina es consistente con lo informado por MINEDUC, quien consigna un aumento de la participación de las mujeres en la matrícula de educación superior, concentrándose en las áreas de Educación, Salud y Ciencias Sociales ${ }^{(12)}$. Asimismo, la edad promedio de los postulantes/año no difiere de los restantes titulados que, según datos del MINEDUC, es de 25,7 años $\pm 0,3$ (25,5 años $\pm 0,3$ en mujeres y 25,7 años $\pm 0,4$ en hombres $)^{(11)}$. Podemos concluir entonces, que los postulantes/año a EDF tienen características similares en términos de edad promedio y proporción hombre/mujer, que los recién titulados.

El promedio de plazas ofrecidas en el período 2009-2018 fue de $38,8 \pm 6,2$, lo que es menor a lo reportado para el período 2003-2009 $(40,5 \pm 5,4)^{(6)}$, manteniéndose constante. Esto difiere de lo observado en los concursos EDF de médico cirujano, en el que claramente existe una tendencia al alza(4). Es necesario especificar que la creación y/o reconversión (cambio de una plaza de EDF odontólogo por una de EDF médico) de plazas, es una atribución exclusiva del Ministerio de Salud(4).

De los valores de offset se debe destacar que el estudio 2003-2009 
señala offsets que variaron entre 1,2 y $1,7^{(6)}$. En esta investigación, durante los últimos tres concursos se han llenado todas las plazas, sin utilizar la lista de espera (valor offset 1,0 ). Sin embargo, durante los años 2014 y 2015 se observaron variaciones (offset 5,3 y 2,0, respectivamente), que coinciden con una polémica gremial entre los cirujanos dentistas de EDF y las autoridades del Ministerio de Salud, razón por la que los dentistas hicieron un llamado a no tomar las plazas $^{(14)}$

Respecto al numero de postulantes a EDF, este representa el 13,8\% del total de egresados pese a que esta participación ha aumentado en comparación al período 2003 a $2009^{(6)}(139,8 \pm 29,3$ versus $164 \pm$ 33,3 en el periodo 2008-2018). Lo anterior, probablemente asociado al aumento de instituciones universitarias que dictan la carrera en el país (tasa de incremento anual de $15,19 \%$ entre el 2000 y el 2009) (13). Debiese ser campo de futuras investigaciones profundizar en las condicionantes que favorecen o disminuyen las postulaciones, pues se observa que el $63 \%$ de las postulaciones se concentra en sólo 5 instituciones y entre ellas, concentran el $86 \%$ de las plazas adjudicadas. Estas instituciones, en orden decreciente de participación, son: Universidad de Concepción, Universidad de Chile, Universidad del Desarrollo, Universidad San Sebastián y Universidad de La Frontera. Este orden constituye un cambio en relación al periodo 2003-2009, en el cual, las universidades con mayor presencia eran las Universidad de Concepción y la Universidad de La Frontera ${ }^{(6)}$.

Por otra parte, este equipo investigador se encuentra actualmente analizando las causales que condicionan la existencia de altas tasas de postulaciones y adjudicaciones de plazas desde sólo un grupo de universidades en desmedro de otras, como por ejemplo lo observado con la Universidad de Chile, Universidad de Concepción y Universidad de La Frontera. Preliminarmente, esto podría explicarse por la existencia de programas internos que fomentan las postulaciones al concurso, el perfil de egreso de estas instituciones o las características de los alumnos que postulan a dichas casa de estudio. Asimismo, se debe profundizar en las causales de la mayor efectividad de los hombres en la adjudicación de las plazas $(p=0,038)$, así como la existencia de rubros cuya evaluación tiene mayor influencia en el resultado de la postulación, de manera de incentivar a los postulantes a privilegiar actividades que favorecen la efectividad de las postulaciones.

Este estudio ha permitido tener una visión más amplia de cómo se ha comportado el concurso EDF en los últimos 10 años a nivel nacional y nos permite describir el resultado de las postulaciones para el ingreso de cirujano dentistas de la última promoción a la ED del ciclo EDF, entre los años 2009 a 2018. En futuros estudios se deberá analizar con mayor profundidad las razones de los recién titulados para postular y los factores que determinar el éxito de estas postulaciones.

\section{CONFLICTO DE INTERÉS}

Los autores declaran no tener conflictos de interés

Tabla 3: Total de alumnos postulantes por universidad por año

\begin{tabular}{|c|c|c|c|c|c|c|c|c|c|c|c|}
\hline Universidad de origen & 2009 & 2010 & 2011 & 2012 & 2013 & 2014 & 2015 & 2016 & 2017 & 2018 & Total \\
\hline U. de Concepción & 31 & 35 & 15 & 24 & 31 & 33 & 31 & 27 & 17 & 18 & 260 \\
\hline U. de Chile & 12 & 26 & 14 & 23 & 14 & 29 & 32 & 33 & 24 & 16 & 223 \\
\hline U. del Desarrollo & 18 & 13 & 26 & 22 & 19 & 26 & 18 & 27 & 18 & 25 & 212 \\
\hline U. San Sebastián & 22 & 22 & 12 & 25 & 6 & 9 & 31 & 22 & 23 & 20 & 191 \\
\hline U. de La Frontera & 33 & 22 & 16 & 18 & 15 & 19 & 29 & 14 & 6 & 9 & 184 \\
\hline U. de Valparaíso & 16 & 19 & 14 & 18 & 7 & 13 & 14 & 11 & 2 & 4 & 118 \\
\hline U. Austral de Chile & $\mathrm{n} / \mathrm{a}$ & $\mathrm{n} / \mathrm{a}$ & 2 & 9 & 14 & 27 & 12 & 6 & 8 & 8 & 86 \\
\hline U. de Talca & 12 & 4 & 5 & 9 & 7 & 9 & 12 & 16 & 8 & 5 & 87 \\
\hline U. Mayor & 4 & 9 & 7 & 9 & 4 & 14 & 14 & 6 & 4 & 6 & 78 \\
\hline U. Andrés Bello & 9 & 1 & 5 & 2 & 1 & 0 & 7 & 13 & 10 & 16 & 64 \\
\hline U. Diego Portales & 5 & 12 & 3 & 2 & 9 & 6 & 10 & 2 & 2 & 6 & 56 \\
\hline U. de Antofagasta & 3 & 5 & 3 & 3 & 0 & 8 & 4 & 0 & 5 & 4 & 35 \\
\hline U. de Los Andes & 2 & 1 & 1 & 3 & 9 & 2 & 4 & 3 & 0 & 5 & 30 \\
\hline P .Universidad Católica de Chile & $\mathrm{n} / \mathrm{a}$ & $\mathrm{n} / \mathrm{a}$ & $\mathrm{n} / \mathrm{a}$ & $\mathrm{n} / \mathrm{a}$ & $\mathrm{n} / \mathrm{a}$ & $\mathrm{n} / \mathrm{a}$ & 7 & 2 & 7 & 7 & 23 \\
\hline U. Finis Terrae & 3 & 3 & 3 & 1 & 1 & 1 & 3 & 1 & 2 & 1 & 19 \\
\hline U. Pedro de Valdivia & $\mathrm{n} / \mathrm{a}$ & $\mathrm{n} / \mathrm{a}$ & $\mathrm{n} / \mathrm{a}$ & $\mathrm{n} / \mathrm{a}$ & 0 & 0 & 2 & 2 & 0 & 2 & 6 \\
\hline U. Bolivariana & $\mathrm{n} / \mathrm{a}$ & $\mathrm{n} / \mathrm{a}$ & $\mathrm{n} / \mathrm{a}$ & 3 & 0 & 0 & 1 & 1 & 0 & 0 & 5 \\
\hline U. de Viña del Mar & $\mathrm{n} / \mathrm{a}$ & $\mathrm{n} / \mathrm{a}$ & $\mathrm{n} / \mathrm{a}$ & $\mathrm{n} / \mathrm{a}$ & $\mathrm{n} / \mathrm{a}$ & $\mathrm{n} / \mathrm{a}$ & 0 & 0 & 3 & 1 & 4 \\
\hline U. del Mar & $\mathrm{n} / \mathrm{a}$ & 0 & 0 & 2 & 0 & 1 & 1 & 0 & $\mathrm{n} / \mathrm{a}$ & $\mathrm{n} / \mathrm{a}$ & 4 \\
\hline U. Extranjeras & 1 & 0 & 0 & 0 & 0 & 0 & 0 & 1 & 0 & 0 & 2 \\
\hline U. Autónoma de Chile & $\mathrm{n} / \mathrm{a}$ & $\mathrm{n} / \mathrm{a}$ & $\mathrm{n} / \mathrm{a}$ & $\mathrm{n} / \mathrm{a}$ & $\mathrm{n} / \mathrm{a}$ & $\mathrm{n} / \mathrm{a}$ & $\mathrm{n} / \mathrm{a}$ & 1 & 0 & 0 & 1 \\
\hline U. Arturo Prat & $\mathrm{n} / \mathrm{a}$ & $\mathrm{n} / \mathrm{a}$ & $\mathrm{n} / \mathrm{a}$ & $\mathrm{n} / \mathrm{a}$ & $\mathrm{n} / \mathrm{a}$ & $\mathrm{n} / \mathrm{a}$ & 0 & 0 & 0 & 1 & 1 \\
\hline Eliminados del análisis & 1 & 1 & 0 & 0 & 0 & 0 & 0 & 0 & 0 & 0 & 2 \\
\hline Total & 172 & 173 & 126 & 174 & 137 & 198 & 232 & 188 & 139 & 154 & 1.691 \\
\hline
\end{tabular}




\section{Bibliografía}

1. World Health Organization. Health systems financing: the path to universal coverage [Internet]. Geneva; 2010. [consultado 26/02/2020]. Disponible en: https:// apps.who.int/iris/bitstream/handle/10665/44371/9789241564021_eng.pdf;jsessioni $\mathrm{d}=9$ C8ECB96660400F9B48F73A8D84A6A02? sequence $=1$

2. World Health Organization. Working together for health, The World Health Report 2006. [Internet]. Geneva; 2006. [consultado 26/02/2020] Disponible en: https://www. who.int/whr/2006/whr06 en.pdf.

3. Verma P, Ford JA, Stuart A, Howe A, Everington S, Steel N. A systematic review of strategies to recruit and retain primary care doctors. BMC Health Serv Res. 2016;161:126.

4. Ministerio de Salud. Plan de ingreso, formación y retención de médicos odontólogos y especialistas. Logros y desafíos. Santiago, Chile: Subsecretaría de Redes Asistenciales; 2018. [consultado 27/02/2020] Disponible en: https:// www.minsal.cl/wp-content/uploads/2018/03/Plan-de-formaci\%C3\%B3n-yretenci\%C3\%B3n-de-especialistas.pdf.

5. Ley 19.664: Normas especiales para los profesionales funcionarios que desempeñan cargos de 11, 22, 33 y 44 horas semanales en los establecimientos de los Servicios de Salud. [Internet]. Santiago, Chile: Ministerio de Salud, 2000 Disponible en: https://www.leychile.cl/Navegar?idNorma=155848 [consultado 27/02/2020].

6. Cartes-Velásquez R. Caracterización de los postulantes a dentista general de zona. Int J Odontostomat. 2011;5(1):71-6.

7. Clouet-Huerta DE, González B, Correa K. Especialización médica en Chile:
Tipos, mecanismos y requisitos de postulación. Una actualización de los procesos para los médicos generales. Rev Med Chile. 2017;145(11):1454-62.

8. Dirección de Presupuestos, Ministerio de Hacienda. Resultados Evaluaciones 2018 [Internet]. Santiago, Chile; 2018. [consultado 26/02/2020]. Disponible en: https://www.dipres.gob.cl/598/articles-177385_doc_pdf.pdf

9. Peña S, Ramirez J, Becerra C, Carabantes J, Arteaga O. The Chilean Rural Practitioner Programme: a multidimensional strategy to attract and retain doctors in rural areas. Bull World Health Organ. 2010;88(5):371-8.

10. Meza D. Atracción y retención de recurso humano en salud en zonas rurales: percepción de profesionales. Rev Estud Políticas Públicas. 2017;5:83-102.

11. Mi Futuro [Internet]. Ministerio de Educación; 2019. [consultado 26/02/2020]. Disponible en: www.mifuturo.cl

12. Servicio de Información de Educación Superior, Ministerio de Educación. Informe brechas de género en la educación superior [Internet]. Santiago, Chile: Subsecretaría de Educación Superior; 2017. [consultado 27/02/2020] Disponible en: https://bibliotecadigital.mineduc.cl/bitstream/handle/20.500.12365/1960/mono712. pdf? sequence $=1$ \&isAllowed $=y$.

13. Rolando R, Salamanca J, Aliaga M. Evolución matrícula educación superior de Chile Período 1990 - 2009. [Internet]. Santiago, Chile; 2010. [consultado 27/02/2020] Disponible en: http://www.sies.cl.

14. Vera S. Dentistas en alerta. Contrangulo [Internet]. 2015. [consultado 26/2020] Disponible en: http://www.contraangulo.com/2015/08/a-juicio-de-dr-jaime-acunapresidente.html. 